\title{
Effect of Head Position Angles on the Blood Flow in the Jugular Vein of Giraffes
}

\section{Rogers Omboga Amenya ${ }^{1,2}$, Johanna Kibet Sigey², Geoffrey Moriaso Ole Maloiy ${ }^{3}$, David Mwangi Theuri²}

\author{
${ }^{1}$ Department of Mathematics and Physical Sciences, Dedan Kimathi University of Technology, Nyeri, Kenya \\ ${ }^{2}$ Department of Pure and Applied Mathematics, Jomo Kenyatta University of Agriculture and Technology, Juja, Kenya \\ ${ }^{3}$ Department of Animal Physiology and Biotechnology, University of Nairobi, Nairobi, Kenya \\ Email: romboga2010@gmail.com
}

How to cite this paper: Amenya, R.O., Sigey, J.K., Maloiy, G.M.O. and Theuri, D.M. (2021) Effect of Head Position Angles on the Blood Flow in the Jugular Vein of Giraffes. World Journal of Mechanics, 11, 165-175. https://doi.org/10.4236/wjm.2021.118012

Received: August 3, 2021

Accepted: August 28, 2021

Published: August 31, 2021

Copyright (c) 2021 by author(s) and Scientific Research Publishing Inc. This work is licensed under the Creative Commons Attribution International License (CC BY 4.0).

http://creativecommons.org/licenses/by/4.0/

\section{Open Access}

\begin{abstract}
The study investigated the effect of the angular position of the head on the blood flow in the jugular vein of giraffes. The vein considered is elastic and collapsible such that its cross-sectional area is not uniform. Transmural pressure causes the blood to move along the vein. Mathematical equations describing the flow were developed, and the vein was considered to be inclined at an angle $\phi$ to the horizontal. A finite-difference scheme was used to solve the equations of motion for the flow. The results are presented via relevant tables and plots. Our findings show that a change in the position of the head causes variation in the external pressure, which in turn causes variation in the cross-sectional area of the vein. Moreover, a drop (or increase) in the inertial pressure of the blood may cause the vein to collapse (or distend), which again triggers a change in the pressure.
\end{abstract}

\section{Keywords}

Venous Flow, Hemodynamics, Collapsible Vein, Blood Pressure, Jugular Vein

\section{Introduction and Mathematical Formulation}

Many fluid-conveying vessels in animal bodies are highly elastic, and in most cases, deform substantially as they respond to the traction (i.e., pressure and viscous stress) exerted by the fluid. Therefore, the study of flows in elastic vessels is of considerable interest and importance for many biomedical and bio-mechanical applications [1]. Moreover, it is an extremely challenging fluid-mechanical problem. For instance, the flow of blood to the neck and brain of a standing gi- 
raffe is of particular interest. Although several experiments have been conducted and theoretical explanations been provided, the issue still remains unresolved. [2] studied the physiology and biophysics of fluid circulation in human bodies and found that "it is no harder in the circulation for the blood to flow uphill than downhill" and that "differences in the level of different parts of the vascular bed do not in any way affect the forces for the flow and hence do not affect directly the circulation." Their study also showed that a continuous column of blood in both the arterial and venous limbs of the loop is a prerequisite for the vascular siphon mechanism [3].

Veterinarians use mathematical modeling to explain how blood flows in and out of the brains of upright animals. The internal jugular veins are the primary venous drain for human brain; however, these veins tend to collapse because they are positioned above the heart level [4]. Consequently, cerebral blood flow in the upright posture would be endangered by a high outflow resistance in the case of no alternative cerebral venous outflow pathways. To address this issue, they attempted to provide an insight into the hemodynamics of the jugular vein in giraffes [5] and [6]. This is because the giraffe has an extremely elongated neck, which also helps them overcome competition from other browsers, for example, in the African savanna [7]. The long neck also plays an important role during fights between young males as well as in courtship rituals. The long neck ( $2 \mathrm{~m}$ in length) necessitates physiological adaptations, especially for the cardiovascular and respiratory systems and for thermoregulatory mechanisms. The cardiovascular system of a giraffe consists of a large range of intravascular pressures caused by the gravitational pressure gradient in an upright state. The heart pumps blood, which then flows through the veins and arteries throughout the body. However, the most interesting aspect of this is the flow of blood to the body parts that are located above the heart, for example, the head. The flow is facilitated by the difference between the atmospheric and internal fluid pressures. For the giraffe, the systolic blood pressure ranges between 280 and $350 \mathrm{mmHg}$, while the diastolic blood pressure ranges between 200 and $300 \mathrm{mmHg}$. Conversely, for a domestic mammal, the systolic blood pressure ranges between 125 and $145 \mathrm{mmHg}$, while the diastolic blood pressure ranges between 80 and 90 $\mathrm{mmHg}$. Compared to the giraffe, these values are significantly lower for other domestic animals; this may be attributed to the considerable height of the giraffe. Moreover, this implies that the heart must generate a very high pressure for the blood to reach the head [8]. However, when the right arterial pressure was measured in a standing, sedated giraffe, it was lower than the pressure in a standing column of blood of the same height as the giraffe's neck. The above phenomenon has motivated several experimental studies to better understand the morphology and structure of a giraffe's neck [9]. However, most of these studies have posed more questions than answers. While using both rigid and collapsible tube models, [10] observed that the siphon mechanism still operates within these vertically oriented models even when the descending limb is partly collapsed and flexible. [11] owing to the presence of a continuous column of 
fluid, the partially collapsed descending veins do not interrupt the siphon mechanism. [10] also emphasized the importance of the interaction between the viscous and hydrostatic components during the measurement of pressure in a vessel. A pressure gradient of 13 to $4 \mathrm{mmHg}$ down the jugular veins of a standing giraffe was attributed to this interaction, as shown by [11] and [12]. [3] obtained a pressure gradient of approximately -93 to $-27 \mathrm{mmHg}$ based on the prevailing hydrostatic gradient, which is related to the sum of the gravitational and viscous pressures. Furthermore, more recent studies have shown that the heart only needs to overcome the viscous resistance of the blood vessels but not the weight of the blood pumped to the head of the giraffe [6] [13] [14]. In this work, we present a mathematical model describing a long straight tube that can collapse as the fluid flows through it, and hence has a variable cross-sectional area [15] and [16]. The tube was considered to be elastic and made of a uniform material, and the flow was assumed to be nonconducting. The tube was assumed to be inclined at angle $\phi$ to the horizontal, such that the total distance moved by the fluid was $L \sin \phi$, where $L$ is the length of the tube. The flow was considered to be along the $\mathrm{x}$-axis, and the full time-independent Navier-tokes equation was solved, in which the length of the tube $(L)$ was considered to be much larger than its radius $(r)$, such that $L \gg r$ [17].

\section{Continuity Equation}

In this section, we present the continuity equation, which is also called the mass conservation equation. It is derived from the law of conservation of mass, which states that the mass remains constant in a steady-state flow (i.e., the stored mass in a controlled volume does not change). In a steady flow, the flow rate does not change with time, implying that the inflow into the controlled volume is equal to the outflow. The continuity equation can be written as

$$
\rho \frac{\partial \rho}{\partial t}+\frac{\partial \rho u_{i}}{\partial u_{i}}=0
$$

where $\rho$ is the fluid density, $t$ is time, $u$ is fluid velocity. For an incompressible flow,

$$
\frac{\partial \rho}{\partial t}=0
$$

and thus the continuity equation reduces to

$$
\frac{\partial \rho u_{i}}{\partial u_{i}}=0
$$

which represents the rate of change of volume of a moving fluid element per unit volume.

\section{Momentum Conservation Equation}

The momentum conservation equation is derived from the law of conservation of momentum, which states that the rate of change of momentum in a controlled 
volume is equal to the sum of the momentum flux into the control volume and any external forces acting on the control volume. This implies that the total momentum of a closed system is constant. Thus, the change in momentum of a small volume element of a fluid is equal to the sum of the dissipative viscous forces, pressure gradient, gravity, and other forces acting on the fluid. The general momentum conservation equation can be written in the tensor form as

$$
\rho\left(\frac{\partial u_{i}}{\partial t}\right)+U_{i}\left(\frac{\partial u_{i}}{\partial x_{i}}\right)=\rho f_{i}+\left(\frac{\partial \sigma_{i, j}}{\partial x_{i}}\right),
$$

where $i=1,2,3$ and $j=1,2,3$ are the summation variables along the $x, y$, and $z$ directions, respectively. The term $\rho f_{i}$ represents the body forces acting on the fluid, which in this study are considered to be the pressure and gravitational forces. The first and second terms on the left side of Equation (4) represent the local and convective accelerations, respectively. For the purpose of this study, the momentum equation can be written in the form:

$$
\rho\left(\frac{\partial u}{\partial t}+u \frac{\partial u}{\partial x}+v \frac{\partial u}{\partial x}\right)=-\frac{\partial p}{\partial x}+\rho g z+\mu\left(\frac{\partial^{2} u}{\partial x^{2}}+\frac{\partial^{2} u}{\partial y^{2}}\right),
$$

where $z$ is the distance between the end points of the jugular vein, $\mu$ is blood viscosity, $g$ is the gravitational forces and $x, y$ are space variables. If the origin is fixed, then the muscles exert a force on the vein, which creates an upward pressure. To determine the pressure gradient, the momentum equation was evaluated at the edge of the boundary layer, where $\rho \rightarrow \rho_{\infty}$. When the fluid is in equilibrium, the upward pressure gradient due to the vein muscle is balanced by the downward pressure gradient due to the variation in the fluid density. Thus, we can write

$$
\nabla p=-\frac{\partial p}{\partial x}-g \rho z
$$

The body force term in the momentum equation along the $\mathrm{x}$-axis can be expressed as

$$
\nabla p=-\frac{\partial p}{\partial x}-g \rho z
$$

By using Equation (6), Equation (7) can be expressed as

$$
\nabla p=\rho_{\infty} g-\rho g z .
$$

However,

$$
\rho_{\infty} g-\rho g z=\rho \beta\left(T_{1}-T_{2}\right),
$$

where $\beta=\frac{1}{\rho}\left(\frac{\partial p}{\partial T}\right)_{p}$ is the coefficient of thermal expansion.

By combining Equations (7), (8), and (9), we get

$$
-\nabla p+\rho g z=\rho g(1+z)+\beta\left(T_{1}-T_{2}\right) .
$$

Equation (10) represents the total pressure gradient term in the momentum equation along the $\mathrm{x}$ axis, such that the momentum equation can now be written 


$$
\rho\left(\frac{\partial u}{\partial t}+u \frac{\partial u}{\partial x}+v \frac{\partial u}{\partial y}\right)=\rho g(1+z)+\beta\left(T_{1}-T_{2}\right)+\mu\left(\frac{\partial^{u}}{\partial x^{2}}+\frac{\partial^{2} u}{\partial y^{2}}\right)
$$

The momentum equation governing the flow is nondimensionalized for convenience, such that

$$
X=\frac{x U}{L}, P=\frac{p}{\rho U}, U=\frac{u}{U}, T=\frac{t U}{L}, Y=\frac{y U}{L},
$$

where $U$ is the characteristic velocity and $L$ is the characteristic length of the system. The derivatives of Equation (12) can thus be written as

$$
\frac{\partial U}{\partial T}+U \frac{\partial U}{\partial X}+V_{o} \frac{\partial U}{\partial Y}=\frac{1}{F_{r}}(1+z)+G r_{\theta}+\frac{1}{R_{e}}\left(\frac{\partial^{2} U}{\partial X^{2}}+\frac{\partial^{2} U}{\partial Y^{2}}\right)
$$

The shape of the vein is given by $z=L \sin \phi$, and hence Equation (22) becomes

$$
\frac{\partial U}{\partial T}+U \frac{\partial U}{\partial X}+V_{o} \frac{\partial U}{\partial Y}=\frac{1}{F_{r}}(1+L \sin \phi)+G r_{\theta}+\frac{1}{R_{e}}\left(\frac{\partial^{2} U}{\partial X^{2}}+\frac{\partial^{2} U}{\partial Y^{2}}\right)
$$

Equation ((14)) is used to analyze the effect of inertia on the steady flow in a collapsing vein.

\subsection{Discretization}

The system of nonlinear equations obtained for the flow problem in this work, that is, Equation (14), was solved using the numerical method of finite differences, where the derivatives in the governing equations are replaced by their corresponding finite difference approximations.

Equation (14) was discretized using the central difference approximation for the partial derivatives with respect to space, while the forward difference approximation was used for the partial derivatives with respect to time. Term $\frac{\partial U}{\partial X}$ can be approximated at discrete node points using the Taylor series expansion of $U_{i, j}^{k}$. The Taylor series expansion of $U_{i+1, j}^{k}$ and $U_{i-1, j}^{k}$ can be expressed in terms of $U_{i, j}^{k}$ and its higher-order derivatives as

$$
U_{i+1, j}^{k}=U_{i, j}^{k}+\Delta x U_{i, j}^{\prime k}+\frac{(\Delta x)^{2}}{2 !} U_{i, j}^{\prime \prime k}+\frac{(\Delta x)^{3}}{3 !} U_{i, j}^{\prime \prime \prime}+\cdots
$$

and

$$
U_{i-1, j}^{k}=U_{i, j}^{k}-\Delta x U_{i, j}^{\prime k}+\frac{(\Delta x)^{2}}{2 !} U_{i, j}^{\prime \prime k}-\frac{(\Delta x)^{3}}{3 !} U_{i, j}^{\prime \prime \prime k}+\cdots
$$

By subtracting Equation (15) from Equation (14) we get

$$
U_{i, j}^{\prime k}=\frac{U_{i+1, j}^{k}-U_{i-1, j}^{k}}{2 \Delta x}+\text { H.O.T. }
$$

The addition of Equations (14) and (15) gives 


$$
U_{i, j}^{\prime \prime k}=\frac{U_{i+1, j}^{k}-2 U_{i, j}^{k}+U_{i-1, j}^{k}}{(\Delta x)^{2}}+\text { H.O.T. }
$$

Note that the derivatives in Equations (15)-(17) are with respect to $x$. Similarly, if the derivatives are taken with respect to $y$, the following equations are obtained:

$$
U_{i, j}^{\prime k}=\frac{U_{i, j+1}^{k}-U_{i, j-1}^{k}}{2 \Delta x}+\text { H.O.T }
$$

and

$$
U_{i, j}^{\prime \prime k}=\frac{U_{i, j+1}^{k}-2 U_{i, j}^{k}+U_{i, j-1}^{k}}{(\Delta y)^{2}}+\text { H.O.T. }
$$

The corresponding time derivative can be written as

$$
U_{i+1, j}^{\prime k}=\frac{U_{i, j}^{k+1}-U_{i, j}^{k}}{\Delta t} .
$$

Finally, Equation (14) is discretized using a finite difference scheme on a uniform mesh, which consists of a plane divided into a network of uniform rectangles of width $\Delta x$ and height $\Delta y$, as shown in Figure 1.

After neglecting the higher-order terms and assuming

$$
K=\frac{1}{F_{r}}(1+L \sin \phi)+G r_{\theta}
$$

to obtain after letting let $\Delta x=\Delta y$; then, the equation becomes

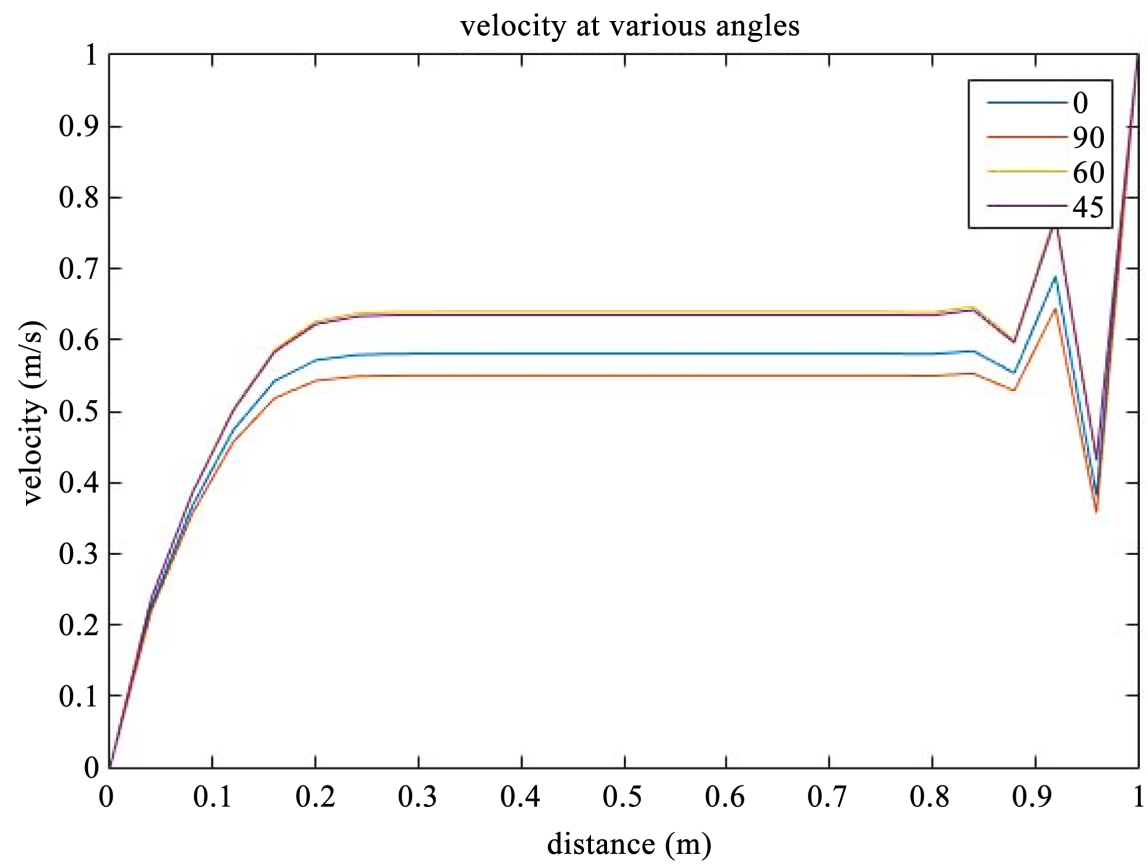

Figure 1. Flow velocity as a function of the distance along the vein at various head position angles, for $T=0.5, M_{x}=25, M_{y}=25, N=10, R_{e}=500, G r=1, F_{r}=10, L=2$, and $v_{o}=0.05$. 


$$
\left\{\begin{array}{l}
r_{1}=\frac{\Delta t}{2 \Delta x} \\
r_{2}=\frac{\Delta t}{2 \Delta y} \\
r=\frac{\Delta t}{R_{e}(\Delta x)^{2}}
\end{array}\right.
$$

to obtain

$$
\begin{aligned}
U_{i, j}^{k+1}= & -r_{1}\left(U_{i+1, j}^{k}-U_{i-1, j}^{k}\right)-V_{0} r_{2}\left(U_{i, j+1}^{k}-U_{i, j-1}^{k}\right)+K \Delta t \\
& +r\left(U_{i+1, j}^{k}-4 U_{i, j}^{k}+U_{i-1, j}^{k}+U_{i, j+1}^{k}+U_{i, j-1}^{k}\right)+U_{i, j}^{k}
\end{aligned}
$$

The boundary conditions used to solve Equation (24) are as follows:

$$
\begin{gathered}
x_{0}, y, t=0=U(x, y, 0), U\left(x_{0}, y, t\right)=0, U\left(x_{f}, y, t\right)=U x_{f}=U x_{0} . \\
U\left(x, y_{0}, T\right)=0=U y_{0}, U\left(x, y_{f}, t\right)=\sin (\pi y)=U y_{f} .
\end{gathered}
$$

\subsection{Results and Discussions}

Equation (24), along with its boundary conditions, was solved using MATLAB to obtain the numerical solutions of the flow variables. According to Figure 2, the velocity of the blood increases up to a distance of $0.2 \mathrm{~m}$ along the vein. Next, the blood flows at a constant velocity up to a distance of $0.875 \mathrm{~m}$, following which the velocity drops slightly before increasing sharply up to a distance of 0.9 $\mathrm{m}$. Subsequently, the velocity increases till discharge. Figure 2 also shows that the velocity of the blood flow depends on the angular position of the head. For instance, we observe that the velocity is higher for more upright head positions.

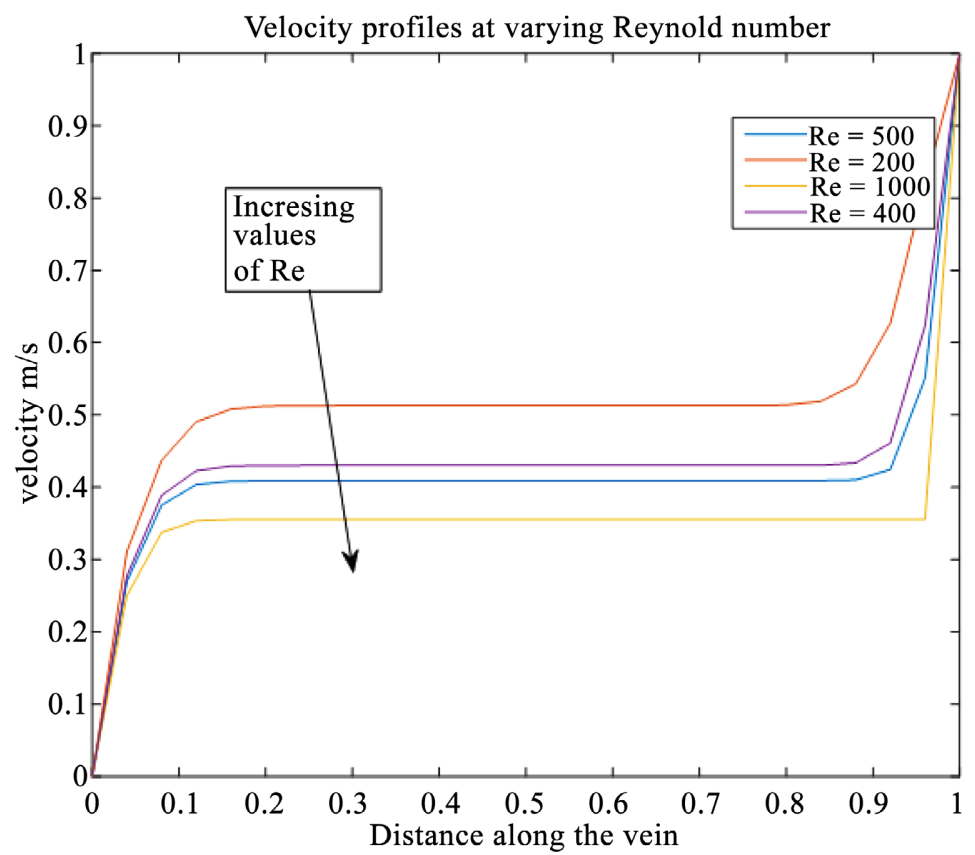

Figure 2. Flow velocity as a function of the distance along the vein at different Re, for $\mu=$ $T=0.5, M_{x}=25, M_{Y}=25, N=10, G r=1, F_{r}=10, L=2, v_{o}=0.05$, and $\phi=45^{\theta}$. 
Figure 3 shows the velocity profiles at different Reynolds numbers (which is defined as the ratio of the inertial forces to viscous forces in the fluid). According to Figure 3, we observe that the velocity increases with decreasing Reynolds number (i.e., with the appearance of viscous forces). The velocity profiles for different Reynolds numbers exhibit the same trend as a function of the distance along the vein. First, the velocity increases sharply with distance, then remains constant, and finally, increases sharply again. The viscous forces between the vein muscles affect the blood as it flows through the vein and consequently retard its motion. Fluctuations in the vein diameter also cause the blood to speed up or slow down accordingly. The Reynolds number was found to be directly proportional to the density and velocity of the blood as well as diameter of the vein. Therefore, increasing the Reynolds number implies increasing the magnitude of any of these three parameters, which can make the blood flow turbulent.

Figure 4 shows the velocity profiles of the blood flow at various angular positions of the head. For all position angles, the velocity of blood starts at zero, then increases sharply until some distance where it slows down, and finally, increases again. For example, when $\phi=90^{\circ}$, the velocity increases from 0 to $0.2 \mathrm{~m} / \mathrm{s}$ for a distance of $0-1.4 \mathrm{~m}$ along the neck, then it increases from 0.2 to $0.4 \mathrm{~m} / \mathrm{s}$ between 1.4 and $1.8 \mathrm{~m}$, and finally, the velocity increases sharply to $0.8 \mathrm{~m} / \mathrm{s}$ between 1.8 and $1.9 \mathrm{~m}$. When $\phi=-45^{\circ}$, the velocity changes from 0 to $0.2 \mathrm{~m} / \mathrm{s}$ within a distance of $0.3 \mathrm{~m}$ along the neck, and then it increases sharply to 0.7 $\mathrm{m} / \mathrm{s}$ within a distance change of $0.1 \mathrm{~m}$, and finally, slows down to $0.8 \mathrm{~m} / \mathrm{s}$ within a further distance change of $0.1 \mathrm{~m}$. When the angle made by the head is zero (i.e., $\phi=0^{\circ}$ ) or the head is in the vertically upright position, the velocity of blood increases from 0 to $0.3 \mathrm{~m} / \mathrm{s}$ within a distance of $1 \mathrm{~m}$, following which it increases significantly to $0.7 \mathrm{~m} / \mathrm{s}$ at a distance of $1.15 \mathrm{~m}$.

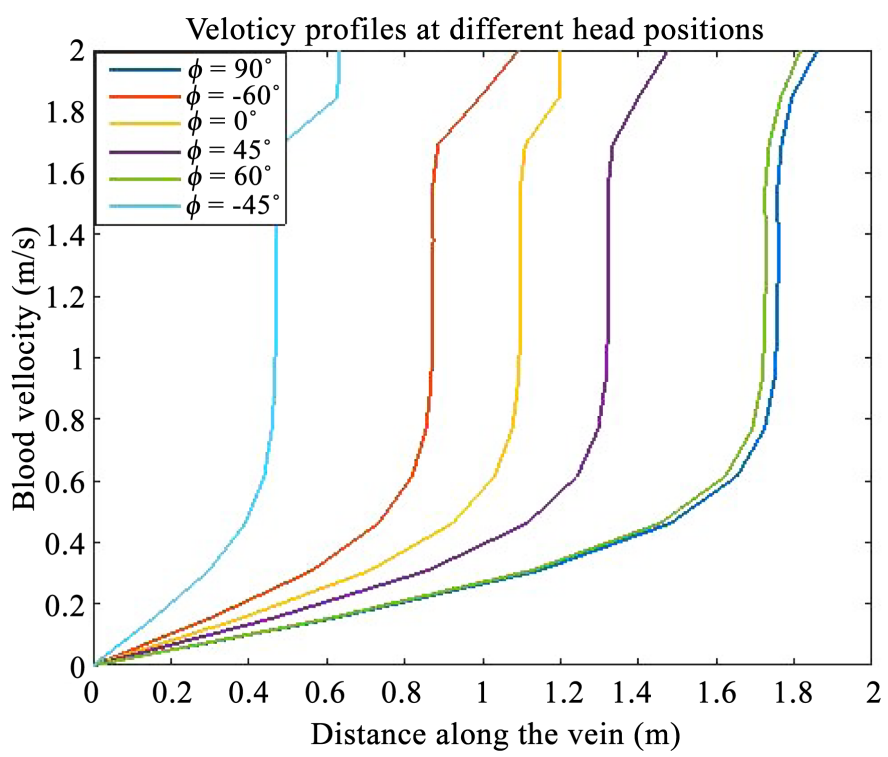

Figure 3. Velocity as a function of the distance along the vein at various head position angles, for $T=0.1, M_{X}=15, M_{Y}=15, N=10, R_{e}=50, G r=10, F_{r}=0.25, \mathrm{~L}=2$, and $v_{0}=$ 0.05 . 


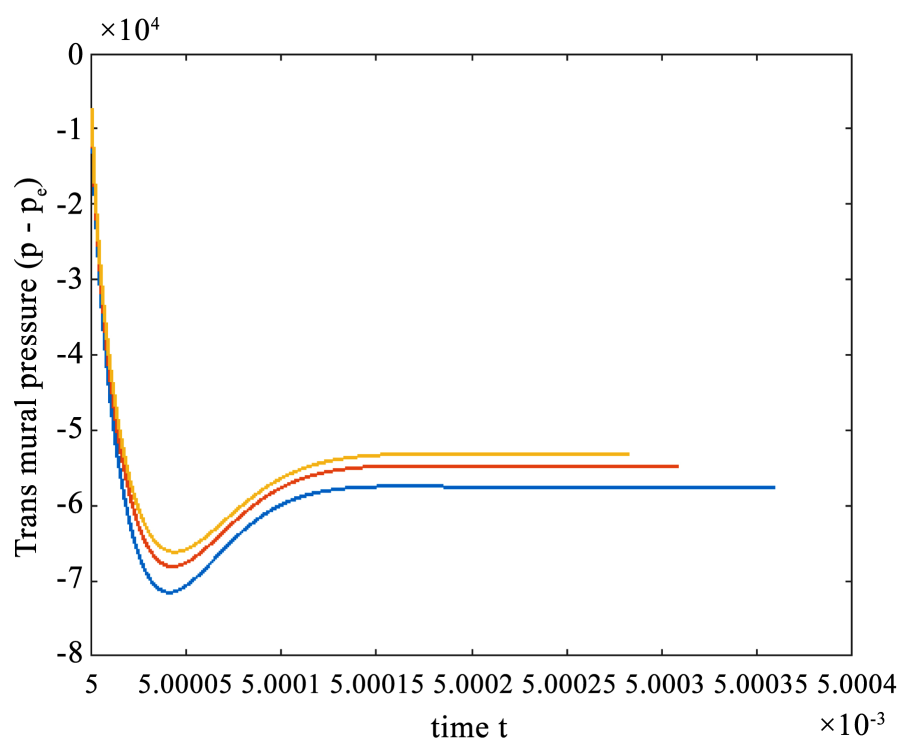

Figure 4. Transmural pressure time along the vein at various head position angles, for $T$ $=0.1, M_{X}=15, M_{Y}=15, N=10, R_{e}=50, G r=10, F_{r}=0.25, L=2$, and $\nu_{0}=0.05$.

According to Figure 4, the blood tends to flow faster as the neck of the giraffe shifts from a vertically downward to a vertically upright position. This is a result of the variation in the gravitational acceleration with time. This also implies that there is a reduction in the cross-sectional area of the vein as the head is raised (i.e., the vein changes from a collapsed to a distended state). This finding confirms the results obtained by Mitchell and Skinner (1993), who showed that the venous flow resistance increases at a constant rate as the head is raised up from a downward position. Brook and Pedley (2002) also showed that the blood flow tends to increase as the giraffe raises its head.

We observe that when the head is at an angle of $90^{\circ}$, the velocity of blood changes slowly with distance along the vein. This implies that when the head is in the horizontal position, the neck muscles relax and the vein assumes an elliptical shape (i.e., the cross-sectional area of the vein is at its maximum), which consequently slows down the blood flow. As the angle decreases further (e.g., $\phi=-45^{\circ}$ ), the velocity starts to increase again see Figure 5.

Figure 5 shows the variation of blood pressure with distance along the vein at different angular positions of the head. We observe that for short distances along the vein, the pressure does not vary significantly, which is true for all head positions. The pressure changes at a larger distance along the vein, and the pressure decreases faster when the angle of inclination of the head is higher. At large values of the transmural pressure, the cross-sectional area of the vein decreases, results in pressure change. The pressure change observed is possibly owing to the stretching of the neck muscle as the head changes positions. This change in head position causes a variation in the external pressure, which in turn causes the cross-sectional area of the vein to expand or contract. Moreover, a drop (or increase) in the inertial pressure of the blood may cause the vein to collapse (or distend), again triggering a change in pressure. 


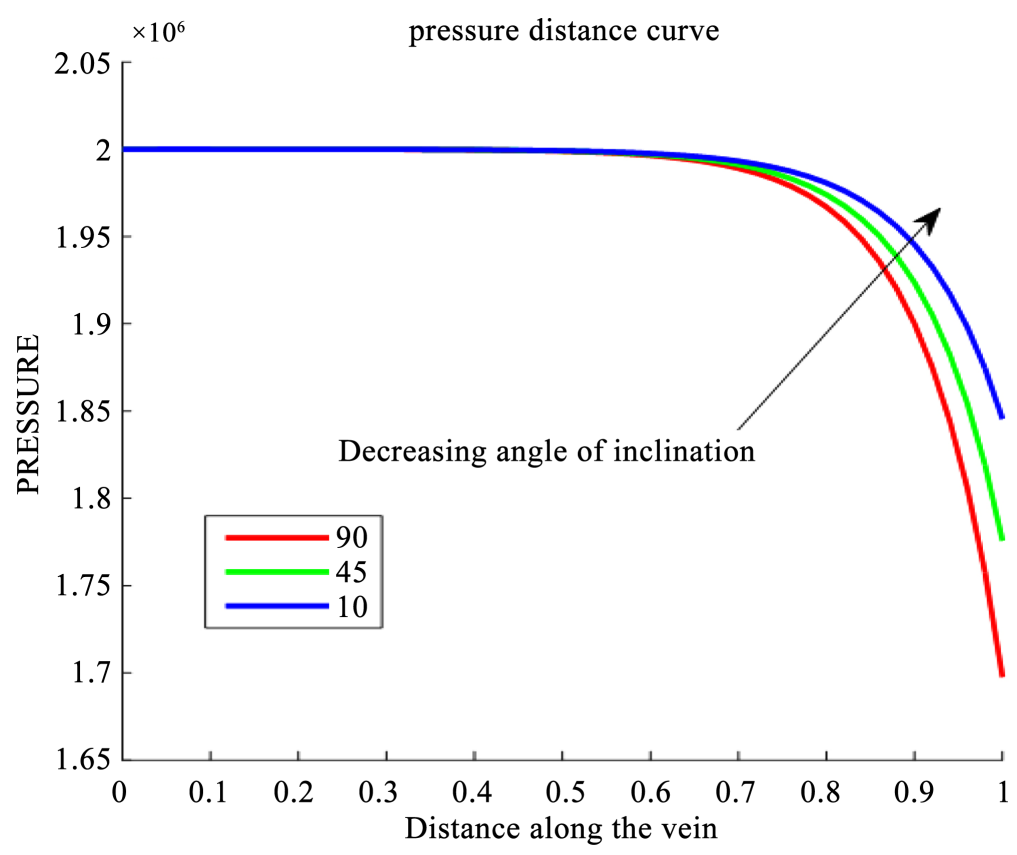

Figure 5. Variation in the pressure as a function of the distance along the vein at different inclination angles of the head with respect to the horizontal, for $G r=500, R_{e}=1000, V_{0}=$ 0.05 , and $F_{r}=8$.

\section{Conclusions}

In this work, we showed that the angular position of the head affects the blood flow in the jugular vein of the giraffe. The following conclusions can be drawn from our study.

1) Blood flow velocity is higher when the head is in an upright position than in the horizontal position.

2) The Reynolds number is inversely proportional to the viscosity of blood. Hence, the blood flow becomes more laminar with increasing viscosity; this in turn decreases the velocity.

3) Conversely, a more viscous and turbulent flow results from a decrease in the blood viscosity. Hemodynamic studies show that any change in the hematocrit value results in a change in the blood viscosity.

4) An increase in the angle of inclination of the head reduces the blood pressure in the vein and vice versa.

\section{Funding}

This study was partially funded by:

1) Higher Education Loans Board (HELB)-Kenya.

2) Dedan Kimathi University of Technology Staff Development fund.

\section{Conflicts of Interest}

The authors declare no conflicts of interest regarding the publication of this paper. 


\section{References}

[1] Ashraf, M.Z. (2018) Mathematical Modelling to Simulate Biological Fluid Flow in a Collapsible Tube. American Journal of Mechanics and Applications, 6.

[2] Hargens, A.R., Millard, R.W., Pettersson, K. and Johansen, K. (1987) Gravitational Haemodynamics and Oedema Prevention in the Giraffe. Nature, 329, 59-60. https://doi.org/10.1038/329059a0

[3] Müller, L.O. and Toro, E.F. (2014) Enhanced Global Mathematical Model for Studying Cerebral Venous Blood Flow. Journal of Biomechanics, 47, 3361-3372. https://doi.org/10.1016/j.jbiomech.2014.08.005

[4] Shapiro, A.H. (1977) Steady Flow in Collapsible Tubes. Journal of Biomechanical Engineering, 99, 126-147. https://doi.org/10.1115/1.3426281

[5] Pedley, T.J. and Luo, X.Y. (1998) Modelling Flow and Oscillations in Collapsible Tubes. Theoretical and Computational Fluid Dynamics, 10, 277-294. https://doi.org/10.1007/s001620050064

[6] Kozlovsky, P., Zaretsky, U., Jaffa, A.J. and Elad, D. (2014) General Tube Law for Collapsible Thin and Thick-Wall Tubes. Journal of Biomechanics, 47, 2378-2384.

[7] Pedley, T.J., Pihler-Puzovic, D. and Puzovic, P. (2015) Flow and Oscillations in Collapsible Tubes: Physiological Applications and Low-Dimensional Models. Technical Report.

[8] Goetz, R.H., Warren, J.V., Gauer, O.H., Patterson, J.L., Doyle, J.T., Keen, E.N., McGregor, M., Tiller, L.M., Smith, M. and Mance, E. (1960) Circulation of the Giraffe. Circulation Research, 8, 1049-1058. https://doi.org/10.1161/01.RES.8.5.1049

[9] Heil, M. and Hazel, A.L. (2011) Fluid-Structure Interaction in Internal Physiological Flows. Annual Review of Fluid Mechanics, 43, 141-162.

[10] Hicks, J.W. and Badeer, H.S. (1992) Gravity and the Circulation: "Open” vs. "Closed” Systems. The American Journal of Physiology, 262, R725-R732. https://doi.org/10.1152/ajpregu.1992.262.5.R725

[11] Nahar, S., Dubey, B.N. and Windhab, E.J. (2019) Influence of Flowing Fluid Property through an Elastic Tube on Various Deformations along the Tube Length. Physics of Fluids, 31, Article ID: 101905. https://doi.org/10.1063/1.5123182

[12] Gadda, G., Taibi, A., Sisini, F., Gambaccini, M., Zamboni, P. and Ursino, M. (2015) A New Hemodynamic Model for the Study of Cerebral Venous Outflow. American Journal of Physiology-Heart and Circulatory Physiology, 308, H217-H231.

[13] Badeer, H.S. (1985) Elementary Hemodynamic Principles Based on Modified Bernoulli's Equation. The Physiologist, 28, 41-46.

[14] Low, H.T. and Chew, Y.T. (1991) Pressure/Flow Relationships in Collapsible Tubes; Effects of Upstream Pressure Fluctuations. Medical \& Biological Engineering \& Computing, 29, 217-221.

[15] Pedley, T.J., Brook, B.S. and Seym, R.S. (1996) Blood Pressure and Flow Rate in the Giraffe Jugular Vein. Technical Report.

[16] Pontrelli, G. (2000) Blood Flow through a Circular Pipe with an Impulsive Pressure Gradient. Mathematical Models and Methods in Applied Sciences, 10, 187-202. https://doi.org/10.1142/S0218202500000124

[17] Ayyaswamy, P.S. (2016) Introduction to Biofluid Mechanics. Fluid Mechanics, Academic Press, Cambridge, e1-e73.

https://doi.org/10.1016/B978-0-12-405935-1.00016-2 